\title{
On the construction of QED using ERG
}

\author{
H Sonoda \\ Physics Department, Kobe University, Kobe 657-8501, Japan \\ E-mail: hsonoda@kobe-u.ac.jp
}

\begin{abstract}
It has been known for some time that a smooth momentum cutoff is compatible with local gauge symmetries. In this paper we show concretely how to construct QED using the exact renormalization group (ERG). First, we give a new derivation of the Ward identity for the Wilson action using the technique of composite operators. Second, parameterizing the theory by its asymptotic behavior for a large cutoff, we show how to fine-tune the parameters to satisfy the identity. Third, we recast the identity as invariance of the Wilson action under a non-linear BRST transformation.
\end{abstract}

PACS numbers: 11.10.Gh, 11.15.-q, 12.20.-m

Submitted to: J. Phys. A: Math. Gen. 


\section{Introduction}

Ken Wilson introduced the exact renormalization group (ERG) to define continuum limits in quantum field theory. [1] Using a theory with a finite ultraviolet (UV) cutoff, we can construct a continuum limit with the expense of keeping an infinite number of terms in the action. The idea was subsequently applied to perturbation theory by Joe Polchinski. 2] The purpose of this paper is to apply Polchinski's version of ERG to construct QED perturbatively.

Before writing anything about gauge theories, we would like to clarify our terminology. The "action" we have mentioned above is a functional of field variables. The momenta of the fields are restricted to the range below a UV cutoff $\Lambda$. The interaction vertices of the action are obtained by integrating out the fluctuations of fields with momenta larger than $\Lambda$. Hence, the correlation functions calculated with this action contain the physics of all momentum scales. The action with a UV cutoff is often called a "Wilson action" in the literature, and we will follow this practice here even though an "action with a UV cutoff" is perhaps more descriptive. The reason is that we wish to avoid confusion with the "effective action" with an infrared (IR) cutoff $\Lambda$ that consists of the 1PI parts of the vertices of the Wilson action. In the limit $\Lambda \rightarrow 0$, the "effective action" with an IR cutoff reduces to the standard effective action. ERG determines the UV cutoff dependence of the Wilson action or the IR cutoff dependence of the "effective action". Except for this introduction, we will consider only the Wilson action in this paper.

The construction of gauge theories in the framework of ERG has been discussed by various authors. To the author's knowledge the first person who applied ERG systematically to construct (non-abelian) gauge theories is Becchi. [3] (There is an even earlier work by Warr [4], who treated ERG mainly as a method of regularization.) Becchi showed how to construct a BRST invariant Wilson action by introducing sources that generate BRST transformations. Alternatively gauge theories can be formulated for the effective action with an IR cutoff. This was initiated by Ellwanger [5] under the name of the "modified" Ward (or Slavnov-Taylor) identities.

Whether we study a Wilson action with a UV cutoff or an effective action with an IR cutoff, the form of the Ward identities depends on the cutoff $\Lambda$ explicitly. (This is the reason for the adjective "modified.") But ERG guarantees the $\Lambda$ independence of the identities: once the identities are satisfied for some $\Lambda$, it is satisfied for any $\Lambda$. It is this property which simplifies the study of gauge symmetry within the ERG framework.

The first explicit construction of QED using ERG was done in [6]. They computed the effective action with $\Lambda=0$ by solving ERG differential equations starting from a set of initial conditions at a very large cutoff $\Lambda_{0}$. They showed how to fine tune the initial conditions so that the effective action with $\Lambda=0$ satisfies the Ward identities. There, ERG is merely a device for loop calculations, and they did not take advantage of the simplicity of the Ward identities at large $\Lambda$. Except for the original work of Becchi, most later works seem to share the same short-coming: the Ward identities are always 
checked at $\Lambda=0$.

The main purpose of this paper is to construct the Wilson action of QED by satisfying the Ward identities at large UV cutoff $\Lambda$. As advocated by Becchi [3], the Ward identities get simplified at large $\Lambda$, and to take advantage of this we need to parameterize the Wilson action in terms of its asymptotic behavior at large $\Lambda$. Such parameterization scheme has been introduced in refs. [7, 8], and we apply it here to QED.

We organize this paper as follows. In sect. 2, we briefly review Polchinski's ERG differential equation by applying it to QED. In sect. 3 we parameterize the Wilson action in terms of its behaviors at large cutoff. This is an application of the scheme explained in [8]. (The reader unfamiliar with perturbative applications of ERG may find a casual reading of [8] helpful.) In sects. 4 and 5 we give a new derivation of the Ward identities for the Wilson action. The result we obtain is equivalent to what has been derived before by Becchi [3] and Ellwanger [5] and others for YM theories. Our starting point is the Ward identities in the continuum limit of QED. A crucial observation, which has not been emphasized or clearly understood in the previous literature on ERG, is that the continuum limit can be constructed using a Wilson action with any finite UV cutoff. (This is derived for the real scalar theory in appendix A.) Hence, we can rewrite the Ward identities in the continuum limit as the identities for the Wilson action. We will end up with an operator equation (27), an equality between two composite operators. In sect. 6, we explain how we can satisfy (27) by fine tuning the parameters of the theory order by order in loop expansions. We give 1-loop calculations in sect. 7. After

giving brief remarks about how to rewrite the Ward identity (27) as BRST invariance in sect. 8, we conclude the paper in sect. 9. Some detailed calculations are given in appendix B.

Before closing we mention some relevant past works. Besides [6], construction of QED using ERG has been done by others, and we mention only a couple here. In [9, 10] the background field method was applied to scalar QED. More recently, a manifestly gauge invariant formulation has been constructed not only for QED [11] but also for general YM theories. 12]

Throughout the paper we use the euclidean metric.

\section{Polchinski's equation}

The Wilson action of QED is given as the sum of free and interaction parts:

$$
S(\Lambda)=S_{\text {free }}(\Lambda)+S_{\text {int }}(\Lambda)
$$

where

$$
\begin{aligned}
S_{\text {free }}(\Lambda) \equiv & -\frac{1}{2} \int_{k} A_{\mu}(k) A_{\nu}(-k) \frac{1}{K(k / \Lambda)}\left(k^{2} \delta_{\mu \nu}-\left(1-\frac{1}{\xi}\right) k_{\mu} k_{\nu}\right) \\
& -\int_{p} \frac{1}{K(p / \Lambda)} \bar{\psi}(-p)(p+i m) \psi(p)
\end{aligned}
$$


We use a sign convention so that the weight of functional integration is given by $\exp [S(\Lambda)]$ instead of the more usual $\exp [-S(\Lambda)]$. The momentum cutoff $\Lambda$ is introduced via a positive cutoff function $K(x)$, which is 1 for $x^{2}<1$ and decays rapidly (e.g., exponentially) for $x^{2}>1$. The free propagators are given by

$$
\begin{aligned}
\left\langle A_{\mu}(k) A_{\nu}(-k)\right\rangle_{S_{\text {free }}(\Lambda)} & =\frac{K(k / \Lambda)}{k^{2}}\left(\delta_{\mu \nu}-(1-\xi) \frac{k_{\mu} k_{\nu}}{k^{2}}\right) \\
\langle\psi(p) \bar{\psi}(-p)\rangle_{S_{\text {free }}(\Lambda)} & =\frac{K(p / \Lambda)}{p+i m}
\end{aligned}
$$

Throughout the paper we consider only the connected part of the correlation functions. For notational simplicity we omit the overall factor of the delta function for momentum conservation.

We can construct the continuum limit using a Wilson action $S(\Lambda)$ with a finite $\Lambda$. But this comes with a price: we must keep an increasing number of terms in the Wilson action as we go to higher orders in perturbation theory. The $\Lambda$ dependence of the interaction part $S_{\text {int }}(\Lambda)$ is given by Polchinski's ERG differential equation [2]:

$$
\begin{aligned}
-\Lambda \frac{\partial S_{\mathrm{int}}(\Lambda)}{\partial \Lambda}= & \int_{k} \frac{\Delta(k / \Lambda)}{k^{2}}\left(\delta_{\mu \nu}-(1-\xi) \frac{k_{\mu} k_{\nu}}{k^{2}}\right) \\
& \times \frac{1}{2}\left(\frac{\delta S_{\mathrm{int}}(\Lambda)}{\delta A_{\mu}(k)} \frac{\delta S_{\mathrm{int}}(\Lambda)}{\delta A_{\nu}(-k)}+\frac{\delta^{2} S_{\mathrm{int}}(\Lambda)}{\delta A_{\mu}(k) \delta A_{\nu}(-k)}\right) \\
& -\int_{p} \Delta(p / \Lambda) \operatorname{Sp}\left[\frac{1}{\not p+i m}\right. \\
& \left.\times\left\{\frac{\vec{\delta}}{\delta \bar{\psi}(-p)} S_{\mathrm{int}}(\Lambda) \cdot S_{\mathrm{int}}(\Lambda) \frac{\overleftarrow{\delta}}{\delta \psi(p)}+\frac{\vec{\delta}}{\delta \bar{\psi}(-p)} S_{\mathrm{int}}(\Lambda) \frac{\overleftarrow{\delta}}{\delta \psi(p)}\right\}\right]
\end{aligned}
$$

where

$$
\Delta\left(\frac{p}{\Lambda}\right) \equiv \Lambda \frac{\partial}{\partial \Lambda} K\left(\frac{p}{\Lambda}\right)
$$

is a non-negative function which is zero for $p^{2}<\Lambda^{2}$, has an appreciative value only for $p^{2} \sim \Lambda^{2}$, and decays rapidly as $p^{2}$ grows. The minus sign in front of the second integral of (5) is due to the Fermi statistics.

It is common to think that $S(\Lambda)$ describes only the physics of low momentum $p<\Lambda$ correctly. On a close inspection, however, we find that $S(\Lambda)$ contains the physics of all momentum scales. To be precise, the renormalized two-point functions are given by

$$
\begin{gathered}
\left\langle A_{\mu}(-k) A_{\nu}(k)\right\rangle=\frac{1-K(k / \Lambda)^{-1}}{k^{2}}\left(\delta_{\mu \nu}-(1-\xi) \frac{k_{\mu} k_{\nu}}{k^{2}}\right) \\
+\frac{1}{K(k / \Lambda)^{2}}\left\langle A_{\mu}(-k) A_{\nu}(k)\right\rangle_{S(\Lambda)} \\
\langle\psi(p) \bar{\psi}(-p)\rangle=\frac{1-K(p / \Lambda)^{-1}}{p+i m}+\frac{1}{K(p / \Lambda)^{2}}\langle\psi(p) \bar{\psi}(-p)\rangle_{S(\Lambda)}
\end{gathered}
$$

where $\langle\cdots\rangle_{S}$ is the correlation function calculated with $S$. The renormalized higher point functions are more simply given by

$$
\left\langle A_{\mu_{1}}\left(k_{1}\right) \cdots A_{\mu_{M}}\left(k_{M}\right) \psi\left(p_{1}\right) \cdots \psi\left(p_{N}\right) \bar{\psi}\left(-q_{1}\right) \cdots \bar{\psi}\left(-q_{N}\right)\right\rangle
$$


On the construction of $Q E D$ using $E R G$

$$
\begin{aligned}
=\prod_{i=1}^{M} & \frac{1}{K\left(k_{i} / \Lambda\right)} \prod_{i=1}^{N} \frac{1}{K\left(p_{i} / \Lambda\right) K\left(q_{i} / \Lambda\right)} \\
& \cdot\left\langle A_{\mu_{1}}\left(k_{1}\right) \cdots A_{\mu_{M}}\left(k_{M}\right) \psi\left(p_{1}\right) \cdots \psi\left(p_{N}\right) \bar{\psi}\left(-q_{1}\right) \cdots \bar{\psi}\left(-q_{N}\right)\right\rangle_{S(\Lambda)}
\end{aligned}
$$

To make sense of the division by $K(p / \Lambda)$, we assume that $K(p / \Lambda)$ is strictly positive and that it vanishes only in the limit $p^{2} \rightarrow \infty$. As long as the Wilson action $S(\Lambda)$ satisfies (5), the right-hand sides of (7,8,9) are independent of the cutoff $\Lambda$. Since the results (7,8,9) may not be widely known, we derive analogous results for the real scalar field theory in appendix A.

\section{Parameterization}

Let us next discuss how to parameterize the solutions of (5). We are only interested in those solutions corresponding to renormalized theories. (See [7, 8] for a similar analysis for the $\phi^{4}$ theory.) We evaluate the functional derivatives of $S(\Lambda)$ at vanishing fields. The asymptotic behaviors at large $\Lambda$ are obtained by expansions in powers of the electron mass and external momenta:

$$
\begin{aligned}
& \left.\frac{\delta^{2} S_{\mathrm{int}}}{\delta A_{\mu}(k) \delta A_{\nu}(-k)}\right|_{0}=\delta_{\mu \nu}\left\{a_{2}(\ln \Lambda / \mu) \Lambda^{2}\right. \\
& \left.+b_{2}(\ln \Lambda / \mu) m^{2}+c_{2}(\ln \Lambda / \mu) k^{2}\right\}+d_{2}(\ln \Lambda / \mu) k_{\mu} k_{\nu}+\cdots \\
& \left.\frac{\vec{\delta}}{\delta \bar{\psi}(-p)} S_{\operatorname{int}} \frac{\overleftarrow{\delta}}{\delta \psi(p)}\right|_{0}=a_{f}(\ln \Lambda / \mu) p+b_{f}(\ln \Lambda / \mu) i m+\cdots \\
& \left.\frac{\vec{\delta}}{\delta \bar{\psi}(-p-k)} \frac{\delta S}{\delta A_{\mu}(k)} \frac{\overleftarrow{\delta}}{\delta \psi(p)}\right|_{0}=a_{3}(\ln \Lambda / \mu) \gamma_{\mu}+\cdots \\
& \left.\frac{\delta^{4} S}{\delta A_{\alpha}\left(k_{1}\right) \delta A_{\beta}\left(k_{2}\right) \delta A_{\gamma}\left(k_{3}\right) \delta A_{\delta}\left(k_{4}\right)}\right|_{0} \\
& =a_{4}(\ln \Lambda / \mu)\left(\delta_{\alpha \beta} \delta_{\gamma \delta}+\delta_{\alpha \gamma} \delta_{\beta \delta}+\delta_{\alpha \delta} \delta_{\beta \gamma}\right)+\cdots
\end{aligned}
$$

where an arbitrary momentum scale $\mu>0$ is introduced to make $\frac{\Lambda}{\mu}$ dimensionless. The vertical line with subscript 0 is a reminder that the derivative is evaluated at vanishing fields. (The extraction of the asymptotic parts is similar to the $\mathcal{T}$ operation in [3].)

For the renormalizable theories, the dotted parts are suppressed by negative powers of $\Lambda$, hence vanishing in the limit $\Lambda / \mu \rightarrow \infty$. The higher order derivatives have negative dimensions, and vanish also in the limit $\Lambda / \mu \rightarrow \infty$.

In solving (5), the seven functions

$$
\begin{gathered}
b_{2}(\ln \Lambda / \mu), c_{2}(\ln \Lambda / \mu), d_{2}(\ln \Lambda / \mu), \\
a_{f}(\ln \Lambda / \mu), b_{f}(\ln \Lambda / \mu), a_{3}(\ln \Lambda / \mu), a_{4}(\ln \Lambda / \mu)
\end{gathered}
$$

are ambiguous by additive constants. (Note that the quadratically divergent $\Lambda^{2} a_{2}$ has no such ambiguity.) Therefore, in order to specify a unique solution of (5), we must 
introduce seven conditions. To begin, we can adopt the following three normalization conditions:

$$
c_{2}(0)=a_{f}(0)=b_{f}(0)=0
$$

$\left(c_{2}(0)\right.$ normalizes the gauge field, $a_{f}(0)$ the spinor fields, and $b_{f}(0)$ the mass parameter $m$.) It is one of the main purposes of this paper to show how the Ward identities determine the remaining four constants

$$
b_{2}(0), d_{2}(0), a_{3}(0), a_{4}(0)
$$

in terms of the elementary charge $e$, which itself is introduced through the Ward identities.

\section{The Ward identities}

Let us recall the renormalized Ward identities in QED. They have two parts. The first part is the identity for the two-point function of the gauge field:

$$
\frac{1}{\xi} k_{\mu}\left\langle A_{\mu}(-k) A_{\nu}(k)\right\rangle=\frac{k_{\nu}}{k^{2}}
$$

The second part is the Ward identities for the higher-point functions:

$$
\begin{aligned}
& \frac{1}{\xi} k_{\mu}\left\langle A_{\mu}(-k) A_{\mu_{1}}\left(k_{1}\right) \cdots A_{\mu_{M}}\left(k_{M}\right) \psi\left(p_{1}\right) \cdots \psi\left(p_{N}\right) \bar{\psi}\left(-q_{1}\right) \cdots \bar{\psi}\left(-q_{N}\right)\right\rangle \\
& =\frac{e}{k^{2}} \sum_{i=1}^{N}\left[\left\langle A_{\mu_{1}}\left(k_{1}\right) \cdots \psi\left(p_{i}-k\right) \cdots\right\rangle-\left\langle A_{\mu_{1}}\left(k_{1}\right) \cdots \bar{\psi}\left(-q_{i}-k\right) \cdots\right\rangle\right]
\end{aligned}
$$

where $e$ is interpreted as the elementary charge defined at scale $\mu$. The Ward identities can be also written as the conservation law:

$$
\begin{aligned}
& k_{\mu}\left\langle J_{\mu}(-k) A_{\mu_{1}}\left(k_{1}\right) \cdots A_{\mu_{M}}\left(k_{M}\right) \psi\left(p_{1}\right) \cdots \psi\left(p_{N}\right) \bar{\psi}\left(-q_{1}\right) \cdots \bar{\psi}\left(-q_{N}\right)\right\rangle \\
& =e \sum_{i=1}^{N}\left[\left\langle A_{\mu_{1}}\left(k_{1}\right) \cdots \psi\left(p_{i}-k\right) \cdots\right\rangle-\left\langle A_{\mu_{1}}\left(k_{1}\right) \cdots \bar{\psi}\left(-q_{i}-k\right) \cdots\right\rangle\right]
\end{aligned}
$$

where $J_{\mu}$ is the charge current. The goal of this section is to find a concrete expression of $J_{\mu}$ in terms of the interaction action $S_{\text {int }}(\Lambda)$.

We first examine (14). Using (7), we can rewrite (14) as

$$
\frac{1}{\xi} k_{\mu}\left\langle A_{\mu}(-k) A_{\nu}(k)\right\rangle_{S(\Lambda)}=K(k / \Lambda) \frac{k_{\nu}}{k^{2}}
$$

Since

$$
\begin{aligned}
& \left\langle A_{\mu}(-k) A_{\nu}(k)\right\rangle_{S(\Lambda)} \\
& \quad=\frac{K(k / \Lambda)}{k^{2}}\left(\delta_{\mu \alpha}-(1-\xi) \frac{k_{\mu} k_{\alpha}}{k^{2}}\right) \cdot\left(\delta_{\alpha \nu}+\left\langle\frac{\delta S_{\mathrm{int}}}{\delta A_{\alpha}(k)} A_{\nu}(k)\right\rangle_{S(\Lambda)}\right)
\end{aligned}
$$

(see appendix A for an analogous result for the real scalar theory), we can rewrite (17) as

$$
k_{\mu}\left\langle\frac{\delta S_{\mathrm{int}}}{\delta A_{\nu}(k)} A_{\nu}(k)\right\rangle_{S(\Lambda)}=0
$$


Next, we examine (15). Using (9) and

$$
\begin{aligned}
& \left\langle A_{\mu}(-k) A_{\mu_{1}}\left(k_{1}\right) \cdots \psi\left(p_{1}\right) \cdots \bar{\psi}\left(-q_{1}\right) \cdots\right\rangle_{S(\Lambda)} \\
& =\frac{K(k / \Lambda)}{k^{2}}\left(\delta_{\mu \alpha}-(1-\xi) \frac{k_{\mu} k_{\alpha}}{k^{2}}\right) \\
& \quad \cdot\left\langle\frac{\delta S_{\mathrm{int}}}{\delta A_{\alpha}(k)} A_{\mu_{1}}\left(k_{1}\right) \cdots \psi\left(p_{1}\right) \cdots \bar{\psi}\left(-q_{1}\right) \cdots\right\rangle_{S(\Lambda)}
\end{aligned}
$$

(again see appendix A), we can rewrite (15) as

$$
\begin{aligned}
& k_{\mu}\left\langle\frac{\delta S_{\mathrm{int}}}{\delta A_{\mu}(k)} A_{\mu_{1}}\left(k_{1}\right) \cdots A_{\mu_{M}}\left(k_{M}\right) \psi\left(p_{1}\right) \cdots \psi\left(p_{N}\right) \bar{\psi}\left(-q_{1}\right) \cdots \bar{\psi}\left(-q_{N}\right)\right\rangle_{S(\Lambda)} \\
& \quad \times \frac{1}{\prod_{i=1}^{M} K\left(k_{i} / \Lambda\right) \prod_{j=1}^{N} K\left(p_{i} / \Lambda\right) K\left(q_{i} / \Lambda\right)} \\
& =e \sum_{n=1}^{N}\left[\left\langle A_{\mu_{1}}\left(k_{1}\right) \cdots \psi\left(p_{n}-k\right) \cdots\right\rangle-\left\langle A_{\mu_{1}}\left(k_{1}\right) \cdots \bar{\psi}\left(-q_{n}-k\right) \cdots\right\rangle\right]
\end{aligned}
$$

To summarize so far, we can replace (14,15) by (19,21). Note that (19) is a special case $M=1, N=0$ of (21). Hence, (21) for arbitrary $M, N$ is equivalent to (14) 15 .

We now define a vector current by

$$
J_{\mu}(-k) \equiv \frac{\delta S_{\text {int }}(\Lambda)}{\delta A_{\mu}(k)}
$$

This is a composite operator since its correlation functions

$$
\begin{aligned}
& \left\langle J_{\mu}(-k) A_{\mu_{1}}\left(k_{1}\right) \cdots \psi\left(p_{1}\right) \cdots \bar{\psi}\left(-q_{1}\right) \cdots\right\rangle \\
& \equiv\left\langle J_{\mu}(-k) A_{\mu_{1}}\left(k_{1}\right) \cdots \psi\left(p_{1}\right) \cdots \bar{\psi}\left(-q_{1}\right) \cdots\right\rangle_{S(\Lambda)} \\
& \quad \times \frac{1}{\prod_{i=1}^{M} K\left(k_{i} / \Lambda\right) \prod_{j=1}^{N} K\left(p_{j} / \Lambda\right) K\left(q_{j} / \Lambda\right)}
\end{aligned}
$$

are independent of $\Lambda$. (See Appendix A for an explanation on composite operators.) To show this, we differentiate (5) with respect to $A_{\mu}(k)$ to obtain

$$
-\Lambda \frac{\partial}{\partial \Lambda} J_{\mu}(-k)=\mathcal{D} \cdot J_{\mu}(-k)
$$

where $\mathcal{D}$ is a linear differential operator defined by

$$
\begin{aligned}
& \mathcal{D} \cdot \mathcal{F} \equiv \int_{l} \frac{\Delta(l / \Lambda)}{l^{2}}\left(\delta_{\mu \nu}-(1-\xi) \frac{l_{\mu} l_{\nu}}{l^{2}}\right) \cdot\left(\frac{\delta S_{\mathrm{int}}}{\delta A_{\mu}(-l)} \frac{\delta \mathcal{F}}{\delta A_{\nu}(l)}+\frac{1}{2} \frac{\delta^{2} \mathcal{F}}{\delta A_{\mu}(l) \delta A_{\nu}(-l)}\right) \\
&-\int_{p} \Delta(p / \Lambda) \operatorname{Sp} \frac{1}{p+i m}\left\{\frac{\vec{\delta}}{\delta \bar{\psi}(-p)} S_{\mathrm{int}} \cdot \mathcal{F} \frac{\overleftarrow{\delta}}{\delta \psi(p)}\right. \\
&\left.\quad+\frac{\vec{\delta}}{\delta \bar{\psi}(-p)} \mathcal{F} \cdot S_{\mathrm{int}} \frac{\overleftarrow{\delta}}{\delta \psi(p)}+\frac{\vec{\delta}}{\delta \bar{\psi}(-p)} \mathcal{F} \frac{\overleftarrow{\delta}}{\delta \psi(p)}\right\}
\end{aligned}
$$

acting on any functional $\mathcal{F}$. (24) guarantees that the correlation functions (23) are independent of $\Lambda$. Thus, from (21, 23), we can rewrite the Ward identities as (16) where $J_{\mu}$ is defined by (22). (For a discussion of composite operators in $\phi^{4}$ theory, see [8].) 


\section{Ward identity as an operator identity}

In this section we wish to rewrite the Ward identities (16) as a single operator equation. For this purpose we construct a composite operator $\Phi(-k)$ whose correlation functions give the right-hand side of (16):

$$
\begin{aligned}
& \left\langle\Phi(-k) A_{\mu_{1}}\left(k_{1}\right) \cdots A_{\mu_{M}}\left(k_{M}\right) \psi\left(p_{1}\right) \cdots \psi\left(p_{N}\right) \bar{\psi}\left(-q_{1}\right) \cdots \bar{\psi}\left(-q_{N}\right)\right\rangle \\
& =e \sum_{i=1}^{N}\left(\left\langle A_{\mu_{1}}\left(k_{1}\right) \cdots \psi\left(p_{i}-k\right) \cdots\right\rangle-\left\langle A_{\mu_{1}}\left(k_{1}\right) \cdots \bar{\psi}\left(-q_{i}-k\right) \cdots\right\rangle\right)
\end{aligned}
$$

Once we construct $\Phi(-k)$, the Ward identities (16) are equivalent to the single operator equation:

$$
k_{\mu} J_{\mu}(-k)=\Phi(-k)
$$

The construction of $\Phi(-k)$ is essential, but it involves a fair amount of technicality. The derivation will be given in appendix $\mathrm{B}$. There, we show that $\Phi(-k)$ is given by

$$
\begin{aligned}
\Phi(-k) \equiv \int_{p} \operatorname{Sp} U(-p-k, p) \\
\cdot\left\{\frac{\vec{\delta}}{\delta \overline{\psi(-p)}} S \cdot S \frac{\overleftarrow{\delta}}{\delta \psi(p+k)}+\frac{\vec{\delta}}{\delta \bar{\psi}(-p)} S \frac{\overleftarrow{\delta}}{\delta \psi(p+k)}\right\} \\
+e \int_{p}\left[-S \frac{\overleftarrow{\delta}}{\delta \psi(p)} \frac{K(p / \Lambda)}{K((p-k) / \Lambda)} \psi(p-k)\right. \\
\left.\quad+\frac{K(p / \Lambda)}{K((p+k) / \Lambda)} \bar{\psi}(-p-k) \frac{\vec{\delta}}{\delta \bar{\psi}(-p)} S\right]
\end{aligned}
$$

where the matrix $U$ is defined by

$$
\begin{aligned}
& U(-p-k, p) \\
& \equiv e\left[K((p+k) / \Lambda) \frac{1-K(p / \Lambda)}{\not p+i m}-\frac{1-K((p+k) / \Lambda)}{\not p+\not k+i m} K(p / \Lambda)\right]
\end{aligned}
$$

$\Phi$ is a composite operator, satisfying

$$
-\Lambda \frac{\partial}{\partial \Lambda} \Phi(-k)=\mathcal{D} \cdot \Phi(-k)
$$

Hence, if (27) is valid asymptotically for large $\Lambda \gg \mu$, then it is valid for any $\Lambda$. As explained in sect. 1 , this is the most important feature of the Ward identity in ERG. A Ward identity similar to (27) has been obtained for the scalar QED in equation (7) of [10], using the background field formalism.

\section{Fine tuning}

We now show how to satisfy the Ward identity (27) for $\Lambda \gg \mu$ by fixing the four constants (13). 
The asymptotic behavior of $k_{\mu} J_{\mu}$ is easily obtained from the asymptotic behavior of the Wilson action, which is given by $(10 a 10 c 10 d)$ :

$$
\begin{aligned}
& \left.\frac{\delta k_{\mu} J_{\mu}(-k)}{\delta A_{\nu}(-k)}\right|_{0}=k_{\nu}\left\{a_{2}(\ln \Lambda / \mu) \Lambda^{2}\right. \\
& \left.+b_{2}(\ln \Lambda / \mu) m^{2}+\left(c_{2}+d_{2}\right)(\ln \Lambda / \mu) k^{2}\right\}+\cdots \\
& \left.\frac{\vec{\delta}}{\delta \bar{\psi}(-p-k)} k_{\mu} J_{\mu}(-k) \frac{\overleftarrow{\delta}}{\delta \psi(p)}\right|_{0}=a_{3}(\ln \Lambda / \mu) \not k+\cdots \\
& \left.\frac{\delta^{3} k_{\mu} J_{\mu}(-k)}{\delta A_{\alpha}\left(k_{1}\right) \delta A_{\beta}\left(k_{2}\right) \delta A_{\gamma}\left(k_{3}\right)}\right|_{0} \\
& =a_{4}(\ln \Lambda / \mu)\left(k_{\alpha} \delta_{\beta \gamma}+k_{\beta} \delta_{\alpha \gamma}+k_{\gamma} \delta_{\alpha \beta}\right)+\cdots
\end{aligned}
$$

The composite operator $\Phi(-k)$ is a dimension 4 scalar operator of momentum $-k$, odd under charge conjugation, and vanishing at $k=0$. Hence, its asymptotic behavior has the same form as above:

$$
\begin{aligned}
& \left.\frac{\delta \Phi}{\delta A_{\nu}(-k)}\right|_{0} \\
& =k_{\nu}\left\{\bar{a}_{2}(\ln \Lambda / \mu) \Lambda^{2}+\bar{b}_{2}(\ln \Lambda / \mu) m^{2}+\bar{d}_{2}(\ln \Lambda / \mu) k^{2}\right\}+\cdots \\
& \left.\frac{\vec{\delta}}{\delta \bar{\psi}(-p-k)} \Phi(-k) \frac{\overleftarrow{\delta}}{\delta \psi(p)}\right|_{0}=\bar{a}_{3}(\ln \Lambda / \mu) \not k+\cdots \\
& \left.\frac{\delta^{3} \Phi(-k)}{\delta A_{\alpha}\left(k_{1}\right) \delta A_{\beta}\left(k_{2}\right) \delta A_{\gamma}\left(k_{3}\right)}\right|_{0} \\
& \quad=\bar{a}_{4}(\ln \Lambda / \mu)\left(k_{\alpha} \delta_{\beta \gamma}+k_{\beta} \delta_{\alpha \gamma}+k_{\gamma} \delta_{\alpha \beta}\right)+\cdots
\end{aligned}
$$

The values of the four dimensionless functions $\bar{b}_{2}, \bar{d}_{2}, \bar{a}_{3}, \bar{a}_{4}$ at $\ln \Lambda / \mu=0$ determine $\Phi(-k)$ uniquely. Therefore, the operator equation (27) is equivalent to the following four equations:

$$
\begin{aligned}
& b_{2}(0)=\bar{b}_{2}(0) \\
& d_{2}(0)=\bar{d}_{2}(0) \\
& a_{3}(0)=\bar{a}_{3}(0) \\
& a_{4}(0)=\bar{a}_{4}(0)
\end{aligned}
$$

where we have used the convention $c_{2}(0)=0$.

Now, $\Phi$ is defined by (28, 29) in terms of the Wilson action $S(\Lambda)$. Therefore, we obtain

$$
\begin{aligned}
& \left.\frac{\delta \Phi}{\delta A_{\nu}(-k)}\right|_{0}=\left.\int_{q} \operatorname{Sp} U(-q-k, q) \frac{\vec{\delta}}{\delta \bar{\psi}(-q)} \frac{\delta S}{\delta A_{\nu}(-k)} \frac{\overleftarrow{\delta}}{\delta \psi(q+k)}\right|_{0} \\
& \left.\frac{\vec{\delta}}{\delta \bar{\psi}(-p-k)} \Phi(-k) \frac{\overleftarrow{\delta}}{\delta \psi(p)}\right|_{0}=e\left(1-a_{f}(\ln \Lambda / \mu)\right) \not k \\
& \quad+\left.\int_{q} \frac{\vec{\delta}}{\delta \bar{\psi}(-p-k)} \operatorname{Sp}\left\{U(-q-k, q) \frac{\vec{\delta}}{\delta \bar{\psi}(-q)} S \frac{\overleftarrow{\delta}}{\delta \psi(q+k)}\right\} \frac{\overleftarrow{\delta}}{\delta \psi(p)}\right|_{0}
\end{aligned}
$$


On the construction of QED using ERG

$$
\begin{aligned}
& \left.\frac{\delta^{3} \Phi(-k)}{\delta A_{\alpha}\left(k_{1}\right) \delta A_{\beta}\left(k_{2}\right) \delta A_{\gamma}\left(k_{3}\right)}\right|_{0} \\
& =\left.\int_{q} \operatorname{Sp} U(-q-k, q) \frac{\vec{\delta}}{\delta \bar{\psi}(-q)} \frac{\delta^{3} S}{\delta A_{\alpha}\left(k_{1}\right) \delta A_{\beta}\left(k_{2}\right) \delta A_{\gamma}\left(k_{3}\right)} \frac{\overleftarrow{\delta}}{\delta \psi(q+k)}\right|_{0}
\end{aligned}
$$

Expanding the right-hand sides in powers of the electron mass and external momenta, we can obtain $\bar{b}_{2}, \bar{d}_{2}, \bar{a}_{3}, \bar{a}_{4}$. On the right-hand sides one extra loop is given explicitly by the integral over $q$, and it is sufficient to know $S(\Lambda)$ only up to $(l-1)$-loop level to determine the four constants $\bar{b}_{2}(0), \bar{d}_{2}(0), \bar{a}_{3}(0), \bar{a}_{4}(0)$ at $l$-loop level. (Note $a_{f}(0)=0$ by convention.) In other words, with $b_{2}(0), d_{2}(0), a_{3}(0), a_{4}(0)$ determined up to $(l-1)$ loop level, we can determine the $l$-loop values of $b_{2}(0), d_{2}(0), a_{3}(0), a_{4}(0)$ by demanding (33). We can thus construct QED using the Ward identities. This recursive nature of the Ward identities has been observed by Becchi [3] and Morris and D'Attanasio [13. among others. We have taken advantage of it using our particular parameterization of the theory.

\section{1-loop calculations}

Using the results of the previous section, let us do 1-loop calculations. We start from the tree-level values:

$$
b_{2}(0)=d_{2}(0)=a_{4}(0)=0, \quad a_{3}(0)=e
$$

At 1-loop, (34a) gives

$$
\left.\frac{\delta \Phi}{\delta A_{\nu}(-k)}\right|_{0}=e^{2} \int_{q} \operatorname{Sp} \gamma_{\nu} U(-q-k, q)
$$

Changing the integration variable as

$$
q \longrightarrow \Lambda q
$$

we obtain

$$
\begin{aligned}
&\left.\frac{\delta \Phi}{\delta A_{\nu}(-k)}\right|_{0}=e^{2} \Lambda^{2} \int_{q} \operatorname{Sp} \gamma_{\nu} \\
& \cdot\left\{K\left(q+\frac{k}{\Lambda}\right) \frac{1-K(q)}{q+i m / \Lambda}-\frac{1-K\left(q+\frac{k}{\Lambda}\right)}{q+\not k / \Lambda+i m / \Lambda} K(q)\right\} \\
& \simeq e^{2} k_{\nu}\left[-2 \Lambda^{2} \int_{q} \frac{1}{q^{2}} \Delta(q)(1-K(q))+\left(m^{2}+\frac{k^{2}}{3}\right) \int_{q} \frac{\Delta(q)}{\left(q^{2}\right)^{2}}\right]
\end{aligned}
$$

where we have expanded the integral in powers of $m / \Lambda$ and $k_{\mu} / \Lambda$. Using

$$
\int_{q} \frac{\Delta(q) K(q)^{n}}{\left(q^{2}\right)^{2}}=\frac{1}{n+1} \int \frac{d^{4} q}{(2 \pi)^{4}} \frac{-2}{q^{2}} \frac{d}{d q^{2}} K(q)^{n+1}=\frac{1}{(4 \pi)^{2}} \frac{2}{n+1}
$$

(independent of the choice of $K$ ), we obtain

$$
b_{2}(0)=\frac{2 e^{2}}{(4 \pi)^{2}}, \quad d_{2}(0)=\frac{2 e^{2}}{3(4 \pi)^{2}}
$$


On the construction of $Q E D$ using $E R G$

Next we consider ( $(34 b)$ at 1-loop:

$$
\begin{aligned}
& \left.\frac{\vec{\delta}}{\delta \bar{\psi}(-p-k)} \Phi(-k) \frac{\overleftarrow{\delta}}{\delta \psi(p)}\right|_{0}-e\left(1-a_{f}(\ln \Lambda / \mu)\right) \not k \\
& =-e^{2} \int_{q} \gamma_{\mu} U(-q-k, q) \gamma_{\nu} \\
& \quad \cdot \frac{1-K((q-p) / \Lambda)}{(q-p)^{2}}\left(\delta_{\mu \nu}-(1-\xi) \frac{(q-p)_{\mu}(q-p)_{\nu}}{(q-p)^{2}}\right) \\
& \simeq-e^{3} \not k \int_{q} \frac{1}{\left(q^{2}\right)^{2}}\left\{\xi K(q)(1-K(q))^{2}+\frac{3-\xi}{4}(1-K(q)) \Delta(q)\right\}
\end{aligned}
$$

where we have used the change of variables (37), and expanded the integral in powers of $k_{\mu} / \Lambda, p_{\mu} / \Lambda$. Hence,

$$
a_{3}(0)-e=-e^{3}\left(\xi \int_{q} \frac{1}{\left(q^{2}\right)^{2}} K(q)(1-K(q))^{2}+\frac{3-\xi}{4(4 \pi)^{2}}\right)
$$

Finally, we consider (34c):

$$
\begin{aligned}
& \left.\frac{\delta^{3} \Phi(-k)}{\delta A_{\alpha}\left(k_{1}\right) \delta A_{\beta}\left(k_{2}\right) \delta A_{\gamma}\left(k_{3}\right)}\right|_{0} \\
& =e^{3} \int_{q} \operatorname{Sp} U(-q-k, q) \\
& \cdot\left\{\gamma_{\gamma} \frac{1-K\left(\left(q-k_{3}\right) / \Lambda\right)}{q-\not k_{3}+i m} \gamma_{\beta} \frac{1-K\left(\left(q-k_{2}-k_{3}\right) / \Lambda\right)}{q-\not k_{2}-\not k_{3}+i m} \gamma_{\alpha}\right. \\
& +(5 \text { permutations })\} \\
& \simeq 2 e^{4}\left(k_{\alpha} \delta_{\beta \gamma}+k_{\beta} \delta_{\gamma \alpha}+k_{\gamma} \delta_{\alpha \beta}\right) \int_{q} \frac{\Delta(q / \Lambda)}{\left(q^{2}\right)^{2}}(1-K(q / \Lambda))^{2}
\end{aligned}
$$

Hence, we obtain

$$
a_{4}(0)=\frac{4}{3} \frac{e^{4}}{(4 \pi)^{2}}
$$

\section{BRST invariance}

To elucidate the gauge structure of QED further, we introduce ghost and antighost fields $c, \bar{c}$. These fields are non-interacting, and we define the total Wilson action by

$$
\bar{S}(\Lambda) \equiv S(\Lambda)-\int_{k} \bar{c}(-k) c(k) \frac{k^{2}}{K(k / \Lambda)}
$$

We now define the BRST transformation as follows:

$$
\begin{aligned}
& \delta_{\epsilon} A_{\mu}(k)=k_{\mu} \epsilon c(k) \\
& \delta_{\epsilon} c(k)=0 \\
& \delta_{\epsilon} \bar{c}(-k)=-\frac{1}{\xi} k_{\mu} A_{\mu}(-k) \epsilon
\end{aligned}
$$


On the construction of $Q E D$ using $E R G$

$$
\begin{aligned}
\delta_{\epsilon} \psi(p)=\int_{k} \epsilon c(k)\left(e \frac{K(p / \Lambda)}{K((p-k) / \Lambda)} \psi(p-k)\right. \\
\left.-U(-p, p-k) \frac{\vec{\delta}}{\delta \bar{\psi}(-p+k)} \bar{S}\right) \\
\delta_{\epsilon} \bar{\psi}(-p)=-\int_{k} \epsilon c(k) e \frac{K(p / \Lambda)}{K((p+k) / \Lambda)} \bar{\psi}(-p-k)
\end{aligned}
$$

where $\epsilon$ is an arbitrary Grassmann variable. The transformation of $\psi$ is highly nonlinear due to the second term. The Ward identity (27) is equivalent to the following BRST invariance:

$$
\delta_{\epsilon} \bar{S}+\int_{k} \epsilon c(k) \int_{p} \operatorname{Sp} U(-p-k, p) \frac{\vec{\delta}}{\delta \bar{\psi}(-p)} \bar{S} \frac{\overleftarrow{\delta}}{\delta \psi(p+k)}=0
$$

The first term is the change of the Wilson action $\bar{S}$ under the transformation. The second term is the change of the fermionic integration measure. Note that the nonvanishing $\delta_{\epsilon} \bar{S}$ implies the non-invariance of the action under the BRST transformation. For example, the non-invariance due to the photon mass term is cancelled precisely by the jacobian.

Due to the electron number conservation, the transformation of $\psi, \bar{\psi}$ under BRST is not unique. Alternatively, we can define the BRST transformation which is symmetric between $\psi$ and $\bar{\psi}$ :

$$
\begin{aligned}
& \delta_{\epsilon} \psi(p)=e K(p / \Lambda) \int_{k} \epsilon c(k) \Psi(p-k) \\
& \delta_{\epsilon} \bar{\psi}(-p)=-e K(p / \Lambda) \int_{k} \epsilon c(k) \bar{\Psi}(-p-k)
\end{aligned}
$$

where

$$
\begin{aligned}
& \Psi(p) \equiv \psi(p)+\frac{1-K(p / \Lambda)}{\not+i m} \frac{\vec{\delta}}{\delta \bar{\psi}(-p)} S_{\mathrm{int}} \\
& \bar{\Psi}(-p) \equiv \bar{\psi}(-p)+S_{\mathrm{int}} \frac{\overleftarrow{\delta}}{\delta \psi(p)} \frac{1-K(p / \Lambda)}{p+i m}
\end{aligned}
$$

are composite operators. The transformations of $A_{\mu}, c, \bar{c}$ are the same as before. The BRST invariance is now given as

$$
\delta_{\epsilon} \bar{S}-\int_{k} \operatorname{Sp}\left[\delta_{\epsilon} \psi(p) \frac{\overleftarrow{\delta}}{\delta \psi(p)}+\frac{\vec{\delta}}{\delta \bar{\psi}(-p)} \delta_{\epsilon} \bar{\psi}(-p)\right]=0
$$

The first form of BRST invariance has been extended further using the antifield formalism in the joint work with Igarashi and Itoh. 14]

\section{Conclusions}

For some time the compatibility of a smooth cutoff with gauge invariance has been established by the previous works $([3],[5,[6,, 9]-[12]$ and references therein) on ERG. Nevertheless, the contrary viewpoint is still prevalent among the practitioners of quantum field theory. A main task of this paper was to introduce a concrete application 
of ERG to convince the reader of the compatibility. In particular we have shown how to construct QED using ERG. The Wilson action with a finite momentum cutoff has exactly the same gauge invariance as the continuum limit. Nothing is compromised. The crucial observation for our construction is that with a Wilson action we can compute the correlation functions in the continuum limit. (See (7 9) and appendix A.)

As we have mentioned in sect. 1, it is some time since the ground work was laid down for incorporation of gauge symmetries in the ERG formalism. As far as formalism goes, Becchi's results in [3] are seminal, if not final. A merit of the present paper is in the use of a particular parameterization of the theory, based on the asymptotic behavior of the Wilson action at a very large cutoff. The Ward identities of the Wilson action simplify for a large cutoff, and we were able to take advantage of it using our parameterization scheme. In a future publication we wish to apply the same scheme to YM theories.

\section{Acknowledgments}

I thank Profs. Y. Igarashi and K. Itoh for the observation that the Ward identity (27) can be interpreted as the BRST invariance (47). Without their help I would have missed the BRST invariance. I also thank Prof. K. Higashijima for a comment which helped me to simplify the presentation of this paper. This work was partially supported by the Grant-In-Aid for Scientific Research from the Ministry of Education, Culture, Sports, Science, and Technology, Japan (\#14340077).

\section{Appendix A. The cutoff dependence of the correlation functions}

The purpose of this appendix is to explain and derive (77,8]9) and (18]201) which play important roles in the main text. For simplicity, we derive the analogous results for the simpler theory of a real scalar field, which is defined by the Wilson action:

$$
S(\Lambda)=S_{\text {free }}(\Lambda)+S_{\text {int }}(\Lambda)
$$

where

$$
\begin{aligned}
& S_{\text {free }}(\Lambda)=-\frac{1}{2} \int_{p} \phi(p) \phi(-p) \frac{p^{2}+m^{2}}{K(p / \Lambda)} \\
& S_{\text {int }}(\Lambda)=\sum_{n=1}^{\infty} \frac{1}{(2 n) !} \int_{p_{1}, \cdots, p_{2 n}} \mathcal{V}_{2 n}\left(\Lambda ; p_{1}, \cdots, p_{2 n}\right) \phi\left(p_{1}\right) \cdots \phi\left(p_{2 n}\right)
\end{aligned}
$$

The interaction part satisfies the following Polchinski differential equation:

$$
-\Lambda \frac{\partial S_{\mathrm{int}}(\Lambda)}{\partial \Lambda}=\int_{p} \frac{\Delta(p / \Lambda)}{p^{2}+m^{2}} \frac{1}{2}\left\{\frac{\delta S_{\mathrm{int}}}{\delta \phi(p)} \frac{\delta S_{\mathrm{int}}}{\delta \phi(-p)}+\frac{\delta^{2} S_{\mathrm{int}}}{\delta \phi(p) \delta \phi(-p)}\right\}
$$

The correlation functions are computed perturbatively using the propagator

$$
\frac{K(p / \Lambda)}{p^{2}+m^{2}}
$$


On the construction of QED using ERG

and the interaction vertices $\mathcal{V}_{2 n}$. We denote the connected part of the $n$-point correlation function as

$$
\begin{aligned}
& \left\langle\phi\left(p_{1}\right) \cdots \phi\left(p_{n}\right)\right\rangle_{S(\Lambda)} \cdot(2 \pi)^{4} \delta^{(4)}\left(p_{1}+\cdots+p_{n}\right) \\
& \quad \equiv \frac{1}{Z} \int[d \phi] \phi\left(p_{1}\right) \cdots \phi\left(p_{n}\right) \mathrm{e}^{S(\Lambda)}
\end{aligned}
$$

where

$$
Z \equiv \int[d \phi] \mathrm{e}^{S(\Lambda)}
$$

Similarly, the correlation functions of a composite operator $\mathcal{O}(p)$ is defined by

$$
\begin{aligned}
& \left\langle\mathcal{O}(p) \phi\left(p_{1}\right) \cdots \phi\left(p_{n}\right)\right\rangle_{S(\Lambda)} \cdot(2 \pi)^{4} \delta^{(4)}\left(p+p_{1}+\cdots+p_{n}\right) \\
& \quad \equiv \frac{1}{Z} \int[d \phi] \mathcal{O}(p) \phi\left(p_{1}\right) \cdots \phi\left(p_{n}\right) \mathrm{e}^{S(\Lambda)}
\end{aligned}
$$

As a preparation, we first prove

$$
\left(-\Lambda \frac{\partial}{\partial \Lambda}+\sum_{i=1}^{n} \frac{\Delta\left(p_{i} / \Lambda\right)}{K\left(p_{i} / \Lambda\right)}\right)\left\langle\mathcal{O}(p) \phi\left(p_{1}\right) \cdots \phi\left(p_{n}\right)\right\rangle_{S(\Lambda)}=0
$$

for any composite operator that satisfies the ERG differential equation

$$
-\Lambda \frac{\partial \mathcal{O}(p)}{\partial \Lambda}=\mathcal{D} \cdot \mathcal{O}(p)
$$

where

$$
\mathcal{D} \equiv \int_{q} \frac{\Delta(q / \Lambda)}{q^{2}+m^{2}}\left(\frac{\delta S_{\mathrm{int}}}{\delta \phi(q)} \frac{\delta}{\delta \phi(-q)}+\frac{1}{2} \frac{\delta^{2}}{\delta \phi(q) \delta \phi(-q)}\right)
$$

(This result was first shown in [3].) (Proof) Using (A.4) and (A.10), we obtain

$$
\begin{aligned}
& -\Lambda \frac{\partial}{\partial \Lambda}\left(\frac{1}{Z} \int[d \phi] \mathcal{O}(p) \phi\left(p_{1}\right) \cdots \phi\left(p_{n}\right) \mathrm{e}^{S(\Lambda)}\right) \\
= & \frac{1}{Z} \int[d \phi] \phi\left(p_{1}\right) \cdots \phi\left(p_{n}\right)\left(\Lambda \frac{\partial \ln Z}{\partial \Lambda} \mathcal{O}(p) \mathrm{e}^{S}-\Lambda \frac{\partial}{\partial \Lambda}\left(\mathcal{O}(p) \mathrm{e}^{S}\right)\right) \\
= & \int_{q} \frac{\Delta(q / \Lambda)}{q^{2}+m^{2}} \frac{1}{Z} \int[d \phi] \phi\left(p_{1}\right) \cdots \phi\left(p_{n}\right) \\
& \times\left[\frac{1}{2} \frac{\delta^{2}}{\delta \phi(-q) \delta \phi(q)}\left(\mathcal{O}(p) \mathrm{e}^{S}\right)+\frac{\delta}{\delta \phi(q)}\left(\phi(q) \frac{q^{2}+m^{2}}{K(q / \Lambda)} \mathcal{O}(p) \mathrm{e}^{S}\right)\right] \\
= & -\sum_{i=1}^{n} \frac{\Delta\left(p_{i} / \Lambda\right)}{K\left(p_{i} / \Lambda\right)} \cdot \frac{1}{Z} \int[d \phi] \mathcal{O}(p) \phi\left(p_{1}\right) \cdots \phi\left(p_{n}\right) \mathrm{e}^{S}
\end{aligned}
$$

which gives (A.9).

From (A.9), we find that

$$
\prod_{i=1}^{n} \frac{1}{K\left(p_{i} / \Lambda\right)} \cdot\left\langle\mathcal{O}(p) \phi\left(p_{1}\right) \cdots \phi\left(p_{n}\right)\right\rangle_{S(\Lambda)}
$$

is independent of the cutoff $\Lambda$.

A particularly important example of a composite operator is

$$
\mathcal{O}(p)=\frac{\delta S_{\text {int }}}{\delta \phi(-p)}
$$


On the construction of $Q E D$ using $E R G$

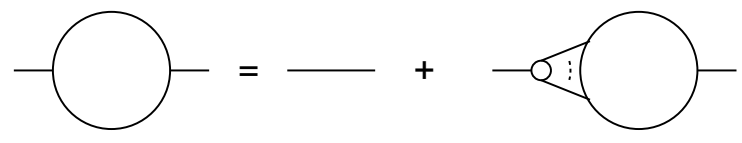

Figure A1. The second graph has at least one interaction vertex.

We can show that $\delta S_{\text {int }} / \delta \phi(-p)$ satisfies (A.10) by differentiating the ERG differential equation (A.4) with respect to $\phi(-p)$.

Now, we are ready. Let us first consider the two-point function. Using the Feynman rules, we immediately find

$$
\langle\phi(p) \phi(-p)\rangle_{S(\Lambda)}=\frac{K(p / \Lambda)}{p^{2}+m^{2}}+\frac{K(p / \Lambda)}{p^{2}+m^{2}}\left\langle\frac{\delta S_{\mathrm{int}}}{\delta \phi(-p)} \phi(-p)\right\rangle_{S(\Lambda)}
$$

The second term denotes the contribution of all the Feynman diagrams with at least one interaction vertex. (See Fig. A1.) This is an analogue of (18). Hence, we obtain

$$
\begin{aligned}
& \frac{1}{K(p / \Lambda)^{2}}\left[\langle\phi(p) \phi(-p)\rangle_{S(\Lambda)}-\frac{K(p / \Lambda)}{p^{2}+m^{2}}\right] \\
& \quad=\frac{1}{K(p / \Lambda)} \frac{1}{p^{2}+m^{2}}\left\langle\frac{\delta S_{\text {int }}}{\delta \phi(-p)} \phi(-p)\right\rangle_{S(\Lambda)}
\end{aligned}
$$

Since $\delta S_{\text {int }} / \delta \phi(-p)$ is a composite operator, this is independent of $\Lambda$. Thus,

$$
\langle\phi(p) \phi(-p)\rangle \equiv \frac{1}{p^{2}+m^{2}}+\frac{1}{K(p / \Lambda)^{2}}\left[\langle\phi(p) \phi(-p)\rangle_{S(\Lambda)}-\frac{K(p / \Lambda)}{p^{2}+m^{2}}\right]
$$

is independent of $\Lambda$. This is the analogue of (778).

For the higher-point correlation functions, there is no graph without interaction vertices. (See Fig. A2.) Hence, we obtain

$$
\left\langle\phi\left(p_{1}\right) \cdots \phi\left(p_{n}\right)\right\rangle_{S(\Lambda)}=\frac{K\left(p_{1} / \Lambda\right)}{p_{1}^{2}+m^{2}}\left\langle\frac{\delta S_{\mathrm{int}}}{\delta \phi\left(-p_{1}\right)} \phi\left(p_{2}\right) \cdots \phi\left(p_{n}\right)\right\rangle_{S(\Lambda)}
$$

for $n>1$. This is an analogue of (20). Since $\delta S_{\text {int }} / \delta \phi\left(-p_{1}\right)$ is a composite operator, we find

$$
\begin{aligned}
& \left.\frac{1}{p_{1}^{2}+m^{2}}\left\langle\frac{\delta S_{\text {int }}}{\delta \phi\left(-p_{1}\right)} \phi\left(p_{2}\right) \cdots \phi\left(p_{n}\right)\right\rangle\right\rangle_{S(\Lambda)} \prod_{i=2}^{n} \frac{1}{K\left(p_{i} / \Lambda\right)} \\
& =\left\langle\phi\left(p_{1}\right) \cdots \phi\left(p_{n}\right)\right\rangle_{S(\Lambda)} \prod_{i=1}^{n} \frac{1}{K\left(p_{i} / \Lambda\right)}
\end{aligned}
$$

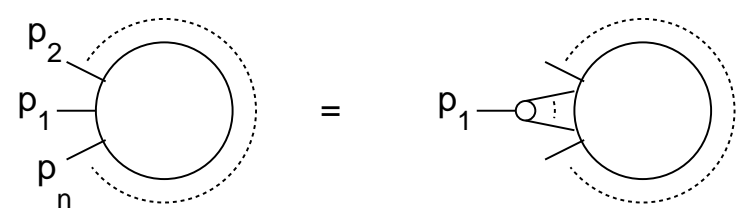

Figure A2. There is at least one interaction vertex for the higher-point correlation functions. 
On the construction of $Q E D$ using $E R G$

is independent of $\Lambda$. This is the analogue of (9).

An alternative derivation of the results given above can be found, for example, in sect. 13 of [1] or in sect. 2 of [14], where more general functional methods with external sources are used.

\section{Appendix B. Construction of $\Phi(-k)$}

The composite operator $\Phi(-k)$, introduced in section 5, is given as the sum of two operators:

$$
\Phi(-k) \equiv \mathcal{O}_{1}(-k)+\mathcal{O}_{2}(-k)
$$

where

$$
\begin{aligned}
\mathcal{O}_{1}(-k) \equiv \int_{p} \operatorname{Sp} U(-p-k, p) & \left\{\frac{\vec{\delta}}{\delta \bar{\psi}(-p)} S \cdot S \frac{\overleftarrow{\delta}}{\delta \psi(p+k)}+\frac{\vec{\delta}}{\delta \bar{\psi}(-p)} S \frac{\overleftarrow{\delta}}{\delta \psi(p+k)}\right\} \\
\mathcal{O}_{2}(-k) \equiv e \int_{p}[ & -S \frac{\overleftarrow{\delta}}{\delta \psi(p)} \frac{K(p / \Lambda)}{K((p-k) / \Lambda)} \psi(p-k) \\
+ & \left.\frac{K(p / \Lambda)}{K((p+k) / \Lambda)} \bar{\psi}(-p-k) \frac{\vec{\delta}}{\delta \bar{\psi}(-p)} S\right]
\end{aligned}
$$

Neither $\mathcal{O}_{1}$ nor $\mathcal{O}_{2}$ are composite operators by themselves. (They do not satisfy $-\Lambda \frac{\partial}{\partial \Lambda} \mathcal{O}_{i}=\mathcal{D} \cdot \mathcal{O}_{i}$.) We wish to compute the correlation functions for $\mathcal{O}_{1}, \mathcal{O}_{2}$ in order to show that $\Phi$ has the desired correlation functions (26).

The operator $\mathcal{O}_{2}(-k)$ is the change of the Wilson action under the following linear change of variables:

$$
\begin{aligned}
\delta \psi(p) & =-e \frac{K(p / \Lambda)}{K((p-k) / \Lambda)} \psi(p-k) \\
\delta \bar{\psi}(-p) & =e \frac{K(p / \Lambda)}{K((p+k) / \Lambda)} \bar{\psi}(-p-k)
\end{aligned}
$$

Since the correlation functions are invariant under linear changes of variables, the correlation functions of $\mathcal{O}_{2}(-k)$ are given by

$$
\begin{aligned}
& \left\langle\mathcal{O}_{2}(-k) A_{\mu_{1}}\left(k_{1}\right) \cdots A_{\mu_{M}}\left(k_{M}\right) \psi\left(p_{1}\right) \cdots \psi\left(p_{N}\right) \bar{\psi}\left(-q_{1}\right) \cdots \bar{\psi}\left(-q_{N}\right)\right\rangle_{S(\Lambda)} \\
& =-\sum_{i=1}^{N}\left[\left\langle A_{\mu_{1}}\left(k_{1}\right) \cdots \delta \psi\left(p_{i}\right) \cdots\right\rangle_{S(\Lambda)}+\left\langle A_{\mu_{1}}\left(k_{1}\right) \cdots \delta \bar{\psi}\left(-q_{i}\right) \cdots\right\rangle_{S(\Lambda)}\right]
\end{aligned}
$$

This gives

$$
\begin{aligned}
& \frac{1}{\prod_{i=1}^{M} K\left(k_{i} / \Lambda\right) \prod_{j=1}^{N} K\left(p_{j} / \Lambda\right) K\left(q_{j} / \Lambda\right)} \\
& \cdot\left\langle\mathcal{O}_{2}(-k) A_{\mu_{1}}\left(k_{1}\right) \cdots A_{\mu_{M}}\left(k_{M}\right) \psi\left(p_{1}\right) \cdots \psi\left(p_{N}\right) \bar{\psi}\left(-q_{1}\right) \cdots \bar{\psi}\left(-q_{N}\right)\right\rangle_{S(\Lambda)} \\
= & e \sum_{i=1}^{N}\left[\left\langle A_{\mu_{1}}\left(k_{1}\right) \cdots \psi\left(p_{i}-k\right) \cdots\right\rangle-\left\langle A_{\mu_{1}}\left(k_{1}\right) \cdots \bar{\psi}\left(-q_{i}-k\right) \cdots\right\rangle\right]
\end{aligned}
$$


On the construction of QED using ERG

for arbitrary $M, N$ except for $M=0, N=1$. In the exceptional case we obtain

$$
\begin{aligned}
& \frac{1}{K((q+k) / \Lambda) K(q / \Lambda)}\left\langle\mathcal{O}_{2}(-k) \psi(q+k) \bar{\psi}(-q)\right\rangle_{S(\Lambda)} \\
& =\frac{e}{K(q / \Lambda)^{2}}\langle\psi(q) \bar{\psi}(-q)\rangle_{S(\Lambda)} \\
& \quad \quad-\frac{e}{K((q+k) / \Lambda)^{2}}\langle\psi(q+k) \bar{\psi}(-q-k)\rangle_{S(\Lambda)} \\
& =\frac{U(-q-k, q)}{K((q+k) / \Lambda) K(q / \Lambda)} \\
& \quad+e(\langle\psi(q) \bar{\psi}(-q)\rangle-\langle\psi(q+k) \bar{\psi}(-q-k)\rangle)
\end{aligned}
$$

where we used (8) in the last step. The first term on the right-hand side is unwanted, and it is the role of $\mathcal{O}_{1}$ to remove it.

Let us compute the correlation functions for $\mathcal{O}_{1}$. For an arbitrary insertion of gauge and $\psi, \bar{\psi}$ fields, we obtain

$$
\int[d A d \psi d \bar{\psi}] \int_{q} \frac{\vec{\delta}}{\delta \bar{\psi}_{i}(-q)}\left(\cdots \mathrm{e}^{S(\Lambda)}\right) \frac{\overleftarrow{\delta}}{\delta \psi_{j}(q+k)} U_{j i}(-q-k, q)=0
$$

This gives

$$
\begin{aligned}
& \left\langle\mathcal{O}_{1}(-k) A_{\mu_{1}}\left(k_{1}\right) \cdots A_{\mu_{M}}\left(k_{M}\right) \psi\left(p_{1}\right) \cdots \psi\left(p_{N}\right) \bar{\psi}\left(-q_{1}\right) \cdots \bar{\psi}\left(-q_{N}\right)\right\rangle_{S(\Lambda)} \\
& -\sum_{i=1}^{N}\left[\left\langle\cdots U\left(-p_{i}, p_{i}-k\right) \frac{\vec{\delta}}{\delta \bar{\psi}\left(-p_{i}+k\right)} S(\Lambda) \cdots\right\rangle_{S(\Lambda)}\right. \\
& \left.\quad+\left\langle\cdots S(\Lambda) \frac{\overleftarrow{\delta}}{\delta \psi\left(q_{i}+k\right)} U\left(-q_{i}-k, q_{i}\right) \cdots\right\rangle_{S(\Lambda)}\right]=0
\end{aligned}
$$

The case $M=0, N=1$ is exceptional because of the inhomogeneity of the equations of motion:

$$
\begin{aligned}
\left\langle U(-q-k, q) \frac{\vec{\delta}}{\delta \bar{\psi}(-p)} S(\Lambda) \cdot \bar{\psi}(-q)\right\rangle_{S(\Lambda)} & =-U(-q-k, q) \\
\left\langle\psi(q+k) \cdot S(\Lambda) \frac{\overleftarrow{\delta}}{\delta \psi(q+k)} U(-q-k, q)\right\rangle_{S(\Lambda)} & =-U(-q-k, q)
\end{aligned}
$$

Hence, we obtain

$$
\left\langle\mathcal{O}_{1}(-k) \psi(q+k) \bar{\psi}(-q)\right\rangle_{S(\Lambda)}=-U(-q-k, q)
$$

Thus, with (B.8) we obtain

$$
\begin{aligned}
& \langle\Phi(-k) \psi(q+k) \bar{\psi}(-q)\rangle \\
& \equiv \frac{1}{K((q+k) / \Lambda) K(q / \Lambda)}\left\langle\left(\mathcal{O}_{1}+\mathcal{O}_{2}\right)(-k) \psi(q+k) \bar{\psi}(-q)\right\rangle_{S(\Lambda)} \\
& =e(\langle\psi(q) \bar{\psi}(-q)\rangle-\langle\psi(q+k) \bar{\psi}(-q-k)\rangle)
\end{aligned}
$$


For the cases other than $M=0, N=1$, the equations of motion are homogeneous:

$$
\begin{aligned}
& \left\langle\cdots U\left(-p_{i}, p_{i}-k\right) \frac{\vec{\delta}}{\delta \bar{\psi}\left(-p_{i}+k\right)} S(\Lambda) \cdots\right\rangle_{S(\Lambda)}=0 \\
& \left\langle\cdots S(\Lambda) \frac{\overleftarrow{\delta}}{\delta \psi\left(q_{i}+k\right)} U\left(-q_{i}-k, q_{i}\right) \cdots\right\rangle_{S(\Lambda)}=0
\end{aligned}
$$

(Note that only the connected parts are considered.) Therefore, we obtain

$$
\left\langle\mathcal{O}_{1}(-k) A_{\mu_{1}}\left(k_{1}\right) \cdots \psi\left(p_{1}\right) \cdots \bar{\psi}\left(-q_{1}\right) \cdots\right\rangle_{S(\Lambda)}=0
$$

Hence, from (B.7) we obtain the desired result

$$
\begin{aligned}
& \left\langle\Phi(-k) A_{\mu_{1}}\left(k_{1}\right) \cdots \psi\left(p_{1}\right) \cdots \bar{\psi}\left(-q_{1}\right) \cdots\right\rangle \\
& =e \sum_{i=1}^{N}\left[\left\langle A_{\mu_{1}}\left(k_{1}\right) \cdots \psi\left(p_{i}-k\right) \cdots\right\rangle-\left\langle A_{\mu_{1}}\left(k_{1}\right) \cdots \bar{\psi}\left(-q_{i}-k\right) \cdots\right\rangle\right]
\end{aligned}
$$

\section{References}

[1] Wilson K and Kogut J 1974 Phys. Rept. C12 75

[2] Polchinski J 1983 Nucl. Phys. B231 269

[3] Becchi C 1991 On the construction of renormalized gauge theories using renormalization group techniques (Parma lectures) Preprint hep-th/9607188

[4] Warr B 1988 Ann. Phys. 183 1; Warr B 1988 Ann. Phys. 18359

[5] Ellwanger U 1994 Phys. Lett. B335 364

[6] Bonini M, D'Attanasio M and Marchesini G 1994 Nucl. Phys. B418 81

[7] Sonoda H 2003 Phys. Rev. D67 065011

[8] Sonoda H 2007 J. Phys. A40 5733

[9] Reuter M and Wetterich C 1994 Nucl. Phys. B427 291

[10] Freire F and Wetterich C 1996 Phys. Lett. B380 337

[11] Arnone S, Morris T and Rosten O 2005 JHEP 0510115

[12] Morris T and Rosten O 2006 J. Phys. A39 11657

[13] Morris T and D'Attanasio M 1996 Phys. Lett. B378 213

[14] Igarashi Y, Itoh K and Sonoda H Quantum master equation for QED in ERG Preprint arXiv:0704.2349 